\title{
1 Case Clinical Report of Cranium Aggressive Fibromatosis and Literature Review
}

\author{
Qi Yan1, Ming Li1, Jintao Li2* $^{*}$ \\ ${ }^{1}$ No. 2 Neurosurgery Department, 1st Affiliated Hospital of Kunming Medical University, Yunnan, China \\ ${ }^{2}$ Neuroscience Institute of Kunming Medical University, Yunnan, China \\ Email: kmyanqi@126.com, lijt8694@126.com,
}

Received 6 July 2015; accepted 12 October 2015; published 15 October 2015

Copyright (C) 2015 by authors and Scientific Research Publishing Inc.

This work is licensed under the Creative Commons Attribution International License (CC BY).

http://creativecommons.org/licenses/by/4.0/

c. (i) Open Access

\section{Abstract}

Objective: The purpose of this study is to explore the clinical characteristics and therapeutic methods of aggressive fibromatosis (AF) in skull. Methods: The clinical data and operative therapy of one case of aggressive fibromatosis in skull in our department were examined and reviewed. The associated literatures were reviewed and discussed. Results: It was indicated that the main manifestation of AF in skull was headache and skull tumor. There was prominent osteolytic destruction found in X-ray plain film for skull AF. And CT scanning showed that skull sclerotin was disorganized and inhomogeneous, with widen diploe. The skull fibromatosis constituted by fibroblasts and myofibroblas, which were mainly spindle-shaped without heteromorphism. Immunohistochemistry showed positive expression of $\beta$-catenin and Vim in these cells. The enlarged incision was adopted for the strategy of operation in this patient of skull AF. After follow-up, there was no recurrence of AF discovered. Conclusions: Skull AF is very rare in neurosurgical clinic. The clinical manifestation and iconography of AF were lack of specificity. Therefore, skull AF is hard to diagnose preoperatively. The effective diagnose is mainly dependant on histopathologic examination. As for treatment, operation is the most optimal method so far, which has a good therapeutic effect.

\section{Keywords}

Skull, Aggressive Fibromatosis, Operation

\section{Introduction}

Aggressive fibromatosis (AF), also named as desmoplastic fibroma (DF), desmoplastic fibroma, is a kind of

${ }^{*}$ Corresponding author. 
scarce tumor of monoclonal fibroblast/myofibroblast. This disease was firstly proposed and named by Muller in 1838. In 1978, Gardini et al. [1] reported the first case of AF in the cranium. In the new category of soft and bone tissue of tumor enacted by WHO, AF was defined as an intermediate tumor that possessed local invasion in the deep part of soft tissues. Herein, we reported a case of aggressive fibromatosis (AF) in cranium who was admitted in the No. 2 Neurosurgery Department of the 2nd Affiliated Hospital of Kunming Medical University. Combined with the available literatures, we preliminarily investigated the characteristics of its manifestation, iconography, diagnosis, differential diagnosis and therapy, with aim to strengthen the recognition for AF in cranium in clinical practice.

\section{Case Report-Cranium Aggressive Fibromatosis Document}

A child patient, fourteen years old, male, was admitted to our department due to a painless masses found in his occiput that was gradually enlarged for 6 months on 10th, February, 2014. Prior to admission, cranium CT of this case in other hospital revealed occipital lesion. This patient did not undergo regular treatment. There was not any cranium trauma history and inheritance history. Medical examination showed that body temperature was $36^{\circ} \mathrm{C}$, pulse was $80 / \mathrm{min}$, with respiration $20 / \mathrm{min}$, blood pressure was $95 / 43 \mathrm{mmHg}$. On the skull of inion region, a $4 \mathrm{~cm} \times 4 \mathrm{~cm}$ sized hard mass was percepted absence of tendemess and positive sign of Nervous System (NS). Laboratory examination showed the homogram, function of blood coagulation and liver, kidney function were all normal. Moreover, the tumor markers, including carcinoembryonic antigen (CEA), cancer antigen (CA), $\alpha$-fetoprotein (AFP) and prostate specific antigen (PSA) were all showed normal.

Cranium CT revealed that the diploe of occipital tuberosity was widened with uneven density. Within the diploe of occipital tuberosity, some sand granule-like pykno-shadows were found. Corresponding endosteal and ectosteal lamella were eroded to be thinned with parts of excalation in sclerotin of ectostel lamella. Adjacent soft tissues were not found to beeroded (Figure 1). Preoperative diagnosis was likely to be vascular tumor in occipital tuberosity. Before operation, a statement on consent to participate under the "ethics, consent and permissions" was signed by this patient. Thereafter, he was subjected to operation of enlarged incision of lesions in occipital tuberosity via central approach on February 19th, 2014. During the operation, we found the periosteum of cranium was integrated. The pathological changes of sclerotin of inion turned out to be off-white fibrous connective tissue-like changes, in which pathological change of the sclerotin was tenacious, the structure of sclerotin was destroyed. As for tumor removal, the excision scope was around $5 \mathrm{~cm} \times 5 \mathrm{~cm}$, the most deepest of lesion tissues approached $4 \mathrm{~cm}$. The structure of endosteal, ectosteal lamella in skull and diploe, which connected tightly with cerebral dura mater, confluence of sinuses and sinus transverses, was found to be destroyed severely. Prior to incision suture, the titanium plate was used to patch up deletion of the occipital bone.

Macropathology of lesion skull tissues removed were off-white in color, $5 \mathrm{~cm} \times 5 \mathrm{~cm} \times 0.8 \mathrm{~cm}$ sized substantive tumor that was tenacious without envelope. The normal structure of bone tissue was destroyed and it was undistinguishable. Microscopy revealed that tumor cells were composed of fusiform cells which were differentiated

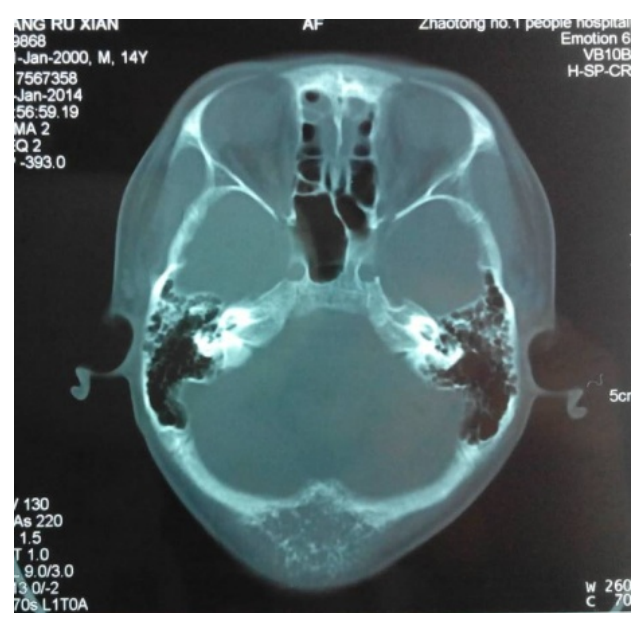

Figure 1. CT manifestations of AF in occipital tuberosity. 
well. These fusiform cells distributed asystematically. Parts of them turned out to be braiding and dermatoglyphic pattern. Under the microscope, local hyaline degeneration was found among which were scattered infiltrative bone tissues that were destroyed. The bone tissues exhibited nodule-like in shape, and were encased by fibrous tissues. The nuclei of bone tissues displayed as oval shape. No heteromorphism phenomenon was discovered (see Figure 2). Showed by immunohistochemisty, $\beta$-catenin $(+)$, Vim (+), Des $(-)$, EMA (-), MSA (-), actin (-), S-100 (-) in lesion bone tissues, with Ki-67 proliferation index 3\% (see Figure 3). The pathological diagnosis of this case of patient was aggressive fibromatosis (AF) in occipital bone. The occipital lump disappeared following the operation. There was no recurrence found when follow-up was conducted until 10 months post of the operation (see Figure 4).

\section{Discussion}

\subsection{Clinical Characterization}

Cranium AF is a kind of rare tumor. Abroad data revealed that its incidence rate occupied around $0.3 \%$ among all benign bone neoplasms, and $0.06 \%$ of all bone neoplasms [2]. The cause of cranium AF is unclear yet. It has

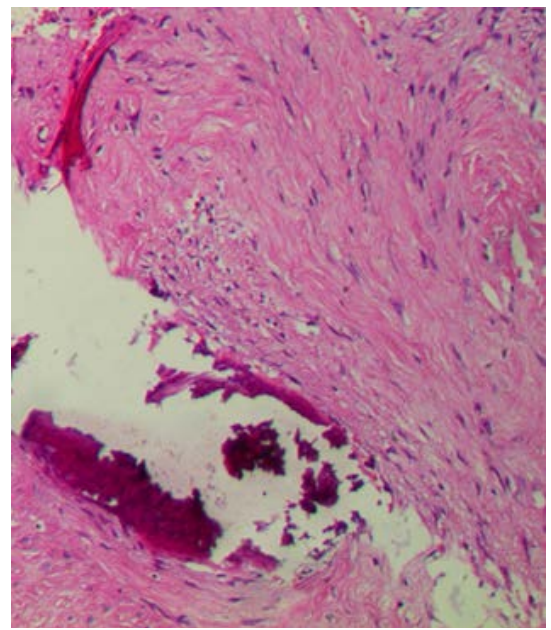

Figure 2. HE staining image showed tumor cells were composed of fusiform cells without nuclueus-mitotic figure. Image showed nodule-like destructive bone tissues $(\mathrm{HE}, \times 200)$.

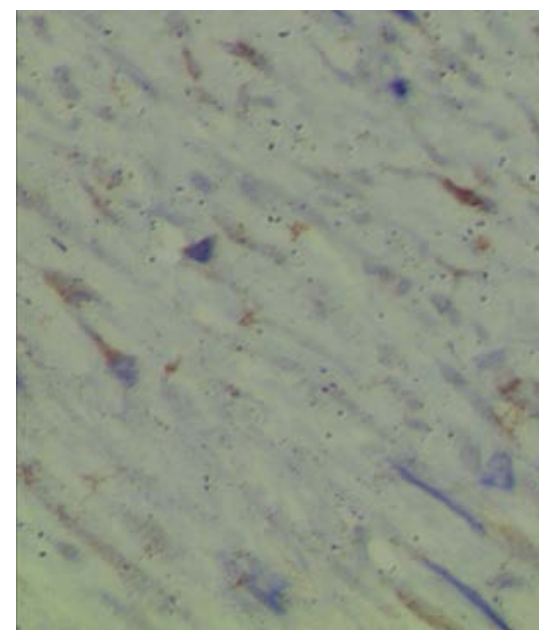

Figure 3. Immunohistochemistry showed tumor cells expressed $\beta$-catenin (SP, $\times 400)$. 


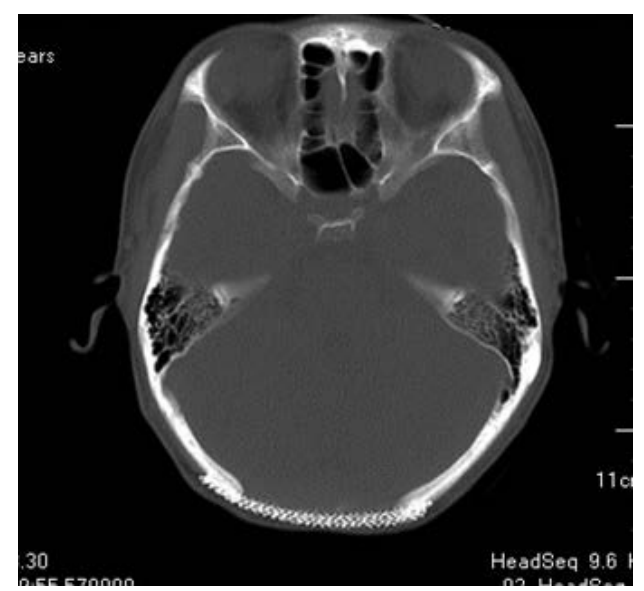

Figure 4. CT image 10 months following operation. There was no recurrence phenomenon found.

been indicated by cellular cytogenetics studies that there exist gene abnormality in the 8th and 20th trisome, the 5th chrosome of APC and $\beta$-cateni [3] in AF patients. There may be correlationship between these genetic abnormality and the incidence and development of AF. AF mainly occurs in muscles of trunk and four limbs, aponeurotic membrane and deep fascia. It is rare that AF implicated to skull [4]-[6]. Up to now, there are 20 abroad literatures involving cranium AF, whereas in domestic, only one case reported by Tang Xiaoping [7]. Among all reports of AF patients, female occupied the major percentage, their ages ranging from child to old people. Their disease course varied from several weeks to decade, with the mean course of half a year. AF most occurs in frontal bone, rarely in occipital bone. The most common manifestation of AF is the skull lump, cranium ostealleosis lay second, impaired vision and hearing decent are rarely occurred. The lesion of AF predominantly happens on unilateral skull. The lesion turned out to be an infiltrative growth style. And the lesion commonly tightly adheres with the cerebral dura mater, it generally dose not break through the cerebral dura mater to invasive the cerebral parenchyma. So far, there are few recurrence of AF reported.

In summary, cranium AF possesses following characteristics: 1) X-ray plain film of skull shows osteolytic destruction in that the lesion could form soft tissue mass, which commonly had a vague boundary to surrounding soft tissues. 2) Plain CT scan reveals that there are some characteristic alterations, including destruction of sclerotin structure, thinningz of endosteal and ectosteal lamella or destruction, widen of diploe, unevenness of bone density. Notably, the enhancement scanning of CT showed reinforcement of focus in common. 3) Lesions in the T1 weighted image of MRI exhibit isointensity and hypointensity, whereas hypointensity in T2 weighted image. There are hyperinsensities found in local areas which are prominently reinforced under enhancement scanning. CT and MRT scan can better reveal the ultra-structure of lesions and sclerotic alterations of endosteal and ectosteal lamella of skull and diploe, which plays a crucial role in ascertaining the scope of tumor boundaries and the invasive extent of surrounding tissues. However, characteristics of iconography above mentioned lack of specificity, various diseases of cranium and meanings have similar iconography manifestation, including CT and MRI [2] [4] [6] [8]-[10]. Among all the cases of cranium AF reported previously, only 3 cases were diagnosed depending on iconography preoperatively, the other cases all diagnosed and confirmed depending on pathological examination post of operation.

Therefore, pathological examination is the most dominant method for the diagnosis of cranium AF. The macropathology features of cranium AF are implicated with off-white in appearance, parenchymatous and tenacious in texture, without envelope. Apart from these, the tangent plane of lesion bone tissues is similar to the glu. Moreover, the adjacent skull tissues are always eroded [11]. Under the microscope, the tumor cells are composed of fibroblasts and myofibroblasts. The tumor tissues are abundant with collagen fibers which interlace together. Local hyaline degeneration was commonly seen within the lesion bone tissues. The AF cells always turn out to be fusiform shape with small and round nuclei, heteromorphism was not found [8] [11]. As for immunophenotype, tumor cells could express Vim or SMA, Des now and then, but not actin and S-100 [9] [10]. Recent studies [12]-[14] found that $\beta$-catenin positive expression had high sensitive in AF diagnosis and differential diagnosis, thereby is considered as a critical index for judgment in diagnosis. In the present clinical study, the pa- 
thological features and immunophenotype of this case of cranium AF are in accordance with the above reports. In addition, research indicated that there existed a great deal of estrogen and progestogen. This notion had not been confirmed in this cranium AF case study [9] [10].

Owing to the lack of specificity in clinical manifestation and iconography in cranium AF, it is vulnerable to be error diagnosed as following diseases. 1) Cranium eosinophilic Granuloma. Both diseases have similar iconographic manifestation. But the latter always has low-grade fever. The macropathology of focus in cranium eosinophilic Granuloma exhibits beige or isabeling flexile granulation tissue, while under the microscope, there are a great amount of Langhans' cells and granulations affluent with blood vessels in the osteolysis destructive area, with a great deal of acidophilic cells. CD1 $\alpha$ and S-100 immunohistochemistry can distinguish these two diseases [11] [15]. 2) Fibrosarcoma of skull: the clinical manisfestation and iconography of this disease is fairly similar to those of cranium AF, but the tumor cells of fibrosarcoma has obvious heteromorphism of nuclei, with prominent miotic figure of Karyokinesis [16]. 3) Fibroblast type of meningioma: this disease has similar manifestation of chinical symptom, iconography, component and morphology of tumor cell with cranium AF. Immunohistochemistry displaying positive EMA expression is the major distinguishable main point [9]. 4) Cranium skeletal angiomatosis, which has a similar manifestation in X plain scan and CT, but MRI T2 weight image showing "black-ringed" that the hypointensity patches containing hemosiderin around the tumor is the most characteristic distinguishable hotspot [17]. 5) Periapical fibroma of cranium: this diseases in commonly implicated to bones apart from the cranium, such as appendicular skeletons. The CT scan displaying ground glass-like is the main characterization to distinguish it with AF [9]. In this study, this patient case did error diagnosed as the periapical fibroma of cranium.

\subsection{Treatment and Prognosis}

So far, operation is the main method to treat cranium AF. Among all the patients cases of cranuum AF had been performed the operation. Due to its local invasive growth style, 16 cases among all cases reported underwent extended extent incision. Following two months to six years' follow-up, recurrence and transferring were not found in even one case of cranium AF patients investigated. It is indicative that extended extent operation, as the preferred therapeutic mode, has a good prognosis in therapy. In clinical practice, parts of cranium patients may belong to circumstances that were inappropriate to perform operation, or experienced operations without radical excision. Another patients encountered recurrence after operation and were not appropriate for operation again. And some patients did not want to receive operative therapy due to other reasons. The latter may need another adjunctive therapy. However, there are few and available effective adjunctive therapeutic strategies reported until now. Owing to its multivariate biologic behavior, AF has a natural cure to a certain extent. The guidelines of Soft Tissue Neoplasms in synthetic tumor National internet of America figured out in 2012 that observation and waiting also seem to an available therapeutic strategy. Therefore, some cranium AF patients may be given clinical close observation. Limited to fewer cases of cranium AF reported, and the fewer years to be followed-up, the recurrence rate of it has not been statistically studied. So, the future clinical research of cranium AF still need cases accumulation and longer-term follow-up. Taken together, cranium AF is rare in clinic. Due to the nonspecific features in its clinical manifestation and iconography, it is hard to pre-operative diagnose for cranium AF. The diagnosis of this disease mainly depends on the histopathilogical examination. The operation is the major therapeutic strategy of cranium of AF. The prognosis of patients who subjected to the operative treatment is relatively good. Feuvret L et al. [18] reported two cases of Chondromyxoid fibroma of the skull incompletely excised lesions treated by postoperative high-dose radiation including proton therapy with no active disease and complication.

\section{Conclusion}

It has been concluded that radiotherapy is not contraindicated for the therapy of this disease. In future clinical research, the radiotherapy for therapy of AF should be applied, and the therapeutic efficiency of it will be intensively studied.

\section{Acknowledgements}

This study was endowed by Yunnan province science and technology project, Grant No. 2015FB008, which is taken charge by Associate Professor Li Jintao. 


\section{References}

[1] Gardini, G., Mingthetti, G. and Ferracini, R. (1978) Desmoplastic Fibroma of Frontal Bone: Report of a Case (Author’s Transl). Pathologica, 70, 575-579.

[2] Rabin, D., Ang, L.C., Megyesi, J., et al. (2003) Desmoplastic Fibroma of the Cranium: Case Report and Review of the Literature. Neurosurgery, 52, 950-954. http://dx.doi.org/10.1227/01.NEU.0000053025.36216.3C

[3] Fletcher, C.D.M., Unni, K.K. and Mertens, F. (2002) World Health Organization Classification of Tumors. Pathology and Genetics of Tumors of Soft Tissue and Bone. IARC Press, Lyon, 83-84.

[4] Cho, B.H., Tye, G.W., Fuller, C.E., et al. (2013) Dsmoplastic Fibroma of the Pediatric Cranium: Case Report and Review of the Literature. Child's Nervous System, 29, 2311-2315. http://dx.doi.org/10.1007/s00381-013-2210-9

[5] Cao, L., Zhao, L.J., Liu, N.B., et al. (2011) The Clinical Characteristics and Prognostic Factors of Aggressive Fibromatosis in 142 Patients. Chinese Journal of Radiation Oncology, 20, 138-141.

[6] Huang, F.-L., Qiu, F.-B., Zhang, X.-F. and Sun, D.-W. (2013) Epidemiologic Feature and Analysis of Diagnosis and Treatment of Desmoids in Recent 20 Years in China. Shandong Medical Journal, 53, 61-63.

[7] Tang, X.-P., Feng, L. and Xie, X.-Y. (2006) 1 Case Report of Skull Desmoids and Literature Review. Chinese Journal of Neurosurgery, 22, 135-137.

[8] Inwards, C.Y., Unni, K.K., Beabout, J.W., et al. (1991) Desmoplastic Fibroma of Bone. Cancer, 67, 1978-1983. http://dx.doi.org/10.1002/1097-0142(19911101)68:9<1978::AID-CNCR2820680922>3.0.CO;2-H

[9] Deniz, K. and Ceylan, D. (2008) Desmoplastic Fibroma of the Skull. Acta Neurochirurgica (Wien), 150, 285-290. http://dx.doi.org/10.1007/s00701-007-1399-3

[10] Wolfe, S.Q., Cervantes, L., Olavarria, G., et al. (2005) Desmoplastic Fibroma of the Pediatric Skull. Report of the Three Cases. Journal of Neurosurgery, 103, 362-365.

[11] Fletcher, C.D.M., Unni, K.K. and Mertens, F. (2002) World Health Organization Classification of Tumors. Pathology and Genetics of Tumors of Soft Tissue and Bone. IARC Press, Lyon, 288.

[12] Bhattacharya, B., Dilworth, H.P., Iacobuzio-Donahue, C., et al. (2005) Muchear Beta-Catenin Expression Distinguishes Deep Fibromatosis from Other Begign and Malignant Fibroblastic and Myofibroblastic Lesions. American Journal of Surgical Pathology, 29, 653-659. http://dx.doi.org/10.1097/01.pas.0000157938.95785.da

[13] Carlson, J.W. and Flether, C.D. (2007) Immunohistochistry for Beta-Catenin in the Differential Diagnosis and of Spindle Cell Lesions: Analysis of a Series and Review of the Literature. Histopathology, 51, 509-514. http://dx.doi.org/10.1111/j.1365-2559.2007.02794.X

[14] Chen, Y.X., Zhang, T. and Shen, D.F. (2013) Role of $\beta$-Catenin in the Diagnosis and Diffential Diagnosis of Fibromatosis. Journal of Clinical and Experimental Pathology, 29, 438-440.

[15] Mammano, S., Candiotto, S. and Balsano, M. (1997) Cast and Brace Treatment of Eosinophilic Granuloma of the Spine. Journal of Pediatric Orthopedics, 17, 821-827. http://dx.doi.org/10.1097/01241398-199711000-00023

[16] Chopra, R., Bhardwaj, M. and Premsagar, I.C. (2010) Fibrosarcoma of the Meninges. Rare Tumors, 2, e3. http://dx.doi.org/10.4081/rt.2010.e3

[17] Haeren, R.H., Dings, J., Hoeberigs, M.C., et al. (2012) Posttraumatic Skull Hemangioma: Case Report. Journal of Neurosurgery, 117, 1082-1088. http://dx.doi.org/10.3171/2012.8.JNS112141

[18] Feuvret, L., Noël, G., Calugaru, V., Terrier, P. and Habrand, J.L. (2005) Chondromyxoid Fibroma of the Skull Base: Differential Diagnosis and Radiotherapy: Two Case Reports and a Review of the Literature. Acta Oncologica, 44, 545553. http://dx.doi.org/10.1080/00365590500237846 